\title{
The myocardial architecture changes in persistent pulmonary hypertension of the newborn in an ovine animal model
}

\author{
Peter Agger 1,2, Satyan Lakshminrusimha ${ }^{3}$, Christoffer Laustsen ${ }^{2,4}$, Sylvia Gugino ${ }^{5}$, Jesper R. Frandsen ${ }^{6}$, Morten Smerup $^{1}$, \\ Robert H. Anderson ${ }^{7}$, Vibeke Hjortdal ${ }^{1,2}$ and Robin H. Steinhorn ${ }^{8}$
}

BACKGROUND: Persistent pulmonary hypertension in the newborn remains a syndrome with high mortality. Knowledge of changes in myocardial architecture in the setting of heart failure in persistent pulmonary hypertension is lacking, and could aid in the explanation of the prevailing high mortality.

METHODS: Persistent pulmonary hypertension was induced by antenatal ligation of the arterial duct in six ovine fetuses. The hearts were compared ex vivo with five matched control hearts, using diffusion tensor imaging to provide the overall anatomical arrangement, and assessment of the angulations and course of the cardiomyocytes. Fibrosis was assessed with histology.

RESULTS: We found an overall increase in heart size in pulmonary hypertension, with myocardial thickening confined to the interventricular septum. An increase of $3.5^{\circ}$ in angulation of myocyte aggregations was found in hypertensive hearts. In addition, we observed a 2.2\% increase in collagen content in the right ventricular free wall. Finally, we found a previously undescribed subepicardial layer of strictly longitudinally oriented cardiomyocytes confined to the right ventricle in all hearts.

CONCLUSION: Myocardial fibrosis and possibly changes in angulations of myocytes seem to play a part in the etiology of persistent pulmonary hypertension. Moreover, a new anatomical arrangement of right ventricular mural architecture is described.

\footnotetext{
P
} ersistent pulmonary hypertension of the newborn is a syndrome of failed circulatory adaptation at birth. It is a lifethreatening condition, often secondary to diverse pathologies such as asphyxia, meconium aspiration syndrome, pneumonia, diaphragmatic hernia, or respiratory distress syndrome. Despite the fact that therapeutic modalities have improved tremendously over the last decades, the syndrome remains a disease with high morbidity and mortality. It is characterized by an elevation of pulmonary vascular resistance, often with normal or low systemic vascular resistance (1). This leads to right-toleft extra-pulmonary shunting, resulting in hypoxemia. The histological changes in the lungs are well described (2). In contrast, its effects on the heart itself are relatively poorly understood, albeit that the notion of congestive heart failure due to elevated right ventricular afterload is relatively common $(2,3)$. Left ventricular dysfunction due to diastolic impairment is also described (4), while a spectrum of myocardial dysfunction was identified in a case-based study (5).

Despite the fact that ventricular mural architecture has been investigated over several centuries, its understanding in the healthy heart remains incomplete. It is hardly surprising, therefore, that an appreciation of the impact of such architecture in the setting of heart failure, or the anatomically abnormal heart, is currently lacking.

Diffusion tensor magnetic resonance imaging has now been used for several years for the purpose of revealing the overall mural architecture. It is a well-validated $(6,7)$ and increasingly used technique to evaluate heart structure (7-9). It depends on the notion that the main direction of spontaneous diffusion of water in the ventricular walls runs parallel to the long axis of the aggregated cardiomyocytes. The main direction of diffusion, thus, becomes a surrogate measure of the main orientation of the cardiomyocytes aggregated together within the myocardial mass.

Multiple studies have pointed to the importance of myocardial remodeling, both throughout the cardiac cycle and in pathology $(9,10)$. To date, however, we are aware of no studies investigating the changes in mural architecture brought upon by persistent pulmonary hypertension of the newborn. Therefore, using a well-established ovine model, we have addressed this problem.

\section{RESULTS}

\section{Anatomical Measurements}

We show the results in terms of anatomy and volumes in Table 1. No difference was found in overall weight between the

\footnotetext{
'Department of Cardiothoracic and Vascular Surgery, Aarhus University Hospital, Aarhus, Denmark; ${ }^{2}$ Department of Clinical Medicine, Aarhus University Hospital, Aarhus, Denmark; ${ }^{3}$ Division of Neonatology, Women and Children's Hospital of Buffalo, State University of New York at Buffalo, Buffalo, New York; ${ }^{4}$ MR Research Centre, Aarhus University Hospital, Aarhus, Denmark; ${ }^{5}$ Department of Physiology and Biophysics, State University of New York at Buffalo, Buffalo, New York; ${ }^{6}$ Center for Functionally Integrative Neuroscience, Aarhus University Hospital, Aarhus, Denmark; ${ }^{7}$ Institute of Genetic Medicine, University of Newcastle, Newcastle-upon-Tyne, UK; ${ }^{8}$ Division of Neonatology, UC Davis Children's Hospital, Sacramento, California. Correspondence: Peter Agger (peter.agger@clin.au.dk)

Received 27 May 2015; accepted 28 September 2015; advance online publication 9 March 2016. doi:10.1038/pr.2015.263
} 
Table 1. Base line assessment

\begin{tabular}{|c|c|c|c|}
\hline & PPHN & Controls & $P$ value \\
\hline Number of hearts $(n)$ & 6 & 5 & \\
\hline Animal weight $(\mathrm{kg})$ & $3.43(0.6)$ & $2.89(0.6)$ & NS \\
\hline Myocardial volume (ml) & $26.83(4.5)$ & $16.91(2.9)$ & 0.0007 \\
\hline \multicolumn{4}{|l|}{ Hemodynamics } \\
\hline Systemic MAP (mmHg) & $42.2(17.5)$ & $56.3(17.1)$ & NS \\
\hline Systemic Oxygenation $(\mathrm{mmHg})$ & $23(12)$ & $263(66)$ & 0.0003 \\
\hline Pulmonary MAP $(\mathrm{mmHg})$ & $39.6(8.1)$ & $35.8(7.7)$ & NS \\
\hline $\begin{array}{l}\text { Pulmonary Blood Flow } \\
(\mathrm{ml} / \mathrm{kg} / \mathrm{min})\end{array}$ & $38(23)$ & $110(55)$ & 0.008 \\
\hline \multicolumn{4}{|l|}{ Left ventricle and septum } \\
\hline Myocardial volume (ml) & $14.8(1.1)$ & $9.8(1.9)$ & 0.004 \\
\hline $\begin{array}{l}\text { Percent of total myocardial } \\
\text { volume (\%) }\end{array}$ & $55(5)$ & $58(4)$ & NS \\
\hline Ventricular volume (ml) & $2.4(1.4)$ & $1.5(0.6)$ & NS \\
\hline Mitral area $\left(\mathrm{mm}^{2}\right)$ & $129.3(44.7)$ & $96.3(19.6)$ & NS \\
\hline Mitral diameter $(\mathrm{cm})$ & $1.2(0.3)$ & $1.1(0.1)$ & NS \\
\hline Mitral circumference $(\mathrm{cm})$ & $4.3(0.7)$ & $3.7(0.5)$ & NS \\
\hline LV wall thickness & $6.1(1.6)$ & $5.9(1.2)$ & $\mathrm{NS}^{\mathrm{R}}$ \\
\hline Septum wall thickness & $7.5(1.8)$ & $6.3(1.3)$ & $0.04^{\mathrm{R}}$ \\
\hline \multicolumn{4}{|l|}{ Right ventricle } \\
\hline Myocardial volume (ml) & $12.1(2.3)$ & $7.1(1.3)$ & 0.0007 \\
\hline $\begin{array}{l}\text { Percent of total myocardial } \\
\text { volume (\%) }\end{array}$ & $45(5)$ & $42(4)$ & NS \\
\hline Ventricular volume (ml) & $3.6(1.8)$ & $1.6(2.0)$ & NS \\
\hline Tricuspid area $\left(\mathrm{mm}^{2}\right)$ & $97.3(18.6)$ & $70.2(33.2)$ & NS \\
\hline Tricuspid diameter $(\mathrm{cm})$ & $1.1(0.1)$ & $0.9(0.2)$ & 0.05 \\
\hline Tricuspid circumference $(\mathrm{cm})$ & $3.9(0.4)$ & $3.1(0.7)$ & 0.03 \\
\hline RV wall thickness & $6.3(1.4)$ & $5.3(1.1)$ & NS \\
\hline
\end{tabular}

Data presented as means (SD). Means compared using Student's $t$-test and repeated measures ANOVA (R)

LV, left ventricle; NS, statistically nonsignificant; PPHN, persistent pulmonary hypertension of the newborn; RV, right ventricle.

six hypertensive animals and their five controls. Myocardial volume was significantly increased, at $26.83 \mathrm{ml}(4.5)$ in the hypertensive animals vs. $16.91 \mathrm{ml}(2.9)$ in controls $(P<0.001)$. Significantly increased myocardial volumes were found in the hypertensive animals for both the combined left ventricle and septum at $14.8 \mathrm{ml}(1.1)$ vs. $9.8 \mathrm{ml}(1.9)$ in controls $(P=0.004)$, and for the right ventricle at $12.1 \mathrm{ml}(2.3)$ vs. $7.1 \mathrm{ml}(1.3)$ in controls $(P=0.0007)$. No differences were found in terms of the ventricular myocardial ratio, or ventricular volumes, but the diameter of the tricuspid valve was significantly increased in the hypertensive hearts at $1.1 \mathrm{~cm}(0.1)$ vs. $0.9 \mathrm{~cm}(0.2)$ in controls $(P=0.05)$, finally the tricuspid circumference was found significantly larger in hypertensive animals at $6.3 \mathrm{~cm}$ (1.4) compared with $5.3(1.1)$ in controls $(P=0.03)$.

Overall, septal thickness was significantly increased by $1.2 \mathrm{~mm}(P=0.04)$, but no significant change in mural thickness was found elsewhere, nor were significant differences found when comparing the left and right ventricular contributions to the total myocardial volume.

\section{Quantitative Measurements of Imaging Data}

The overall difference in helical and transmural angles between hypertensive and normotensive animals was not statistically significant. In Figure 1, we show both positive and negative angulations of all angles for the purpose of comparison to previous studies. As seen, the intrusion and unit angles are evenly distributed around zero. Because we are mainly interested in the absolute change in angulation, we allow ourselves to analyze the data statistically as absolute values as has been done by others (11). The results on unit angulations are summarized in Table 2. An extended version of Table 2 containing regional angle measurements is provided as supplemental data (see Supplementary Table S1 online). An overall statistical significant difference was found between groups $(P<0.001)$. When cumulating all data in the entire heart, there is an overall increase in mean unit angle of $3.5^{\circ}$, at 14.3 in hypertensive animals vs. 12.1 in their controls, $P<0.001$. This increase due to hypertension is roughly equal for all transmural levels. Looking at each ventricle individually, there is an overall increase in the left ventricle of $2.7^{\circ}(P<0.001), 3.5^{\circ}$ in the interventricular septum, and $1.1^{\circ}(P<0.001)$ in the right ventricle. In the left and the right ventricle, equal increases of $\sim 2.5^{\circ}$ were found in the midwall level, while in the subepicardium increases of $3.4^{\circ}$ was found in the left and $1.5^{\circ}$ in the right ventricle. No significant differences were found in the subendocardium. In the septum, an increase of $7.4^{\circ}$ was found in the left ventricular subendocardium, $3.0^{\circ}$ in the midwall level, while no differences were found in the right ventricular subendocardium.

\section{Myocardial Fibrosis}

Results on the fibrosis assessment are shown in Figure 2. In the right ventricle, the collagen content was found to be $3.8 \%$ in hypertensive hearts. This was significantly increased compared with the value of $1.6 \%$ obtained in controls, $P=0.048$. Posthoc analysis revealed that the fibrotic changes were confined to the epicardial third of the transmural thickness. Here, the collagen content was $4.4 \%$, as compared with $1.7 \%$ in controls, $P=0.02$. Using Spearman's correlation, we found no correlation between the ratios of fibrosis in the right ventricle and their corresponding measures of angulation of myocardial aggregates, $\rho_{\mathrm{s}}=0.14, P=0.45$.

\section{Qualitative Findings}

We were surprised to find a strictly longitudinal layer of cardiomyocytes confined strictly to the right ventricular subepicardium of both control and hypertensive hearts (Figure 3). An abrupt transition is present between this superficial right ventricular layer and the deeper parts of the wall, the latter showing a more classical distribution of helical angles. The phenomenon is revealed by the abrupt change in the plotted curve of helical angles, most obvious in zone r5 (Figure 3). Histological haematoxylin-eosin sections confirmed the findings provided by magnetic resonance imaging (Figure 4). 


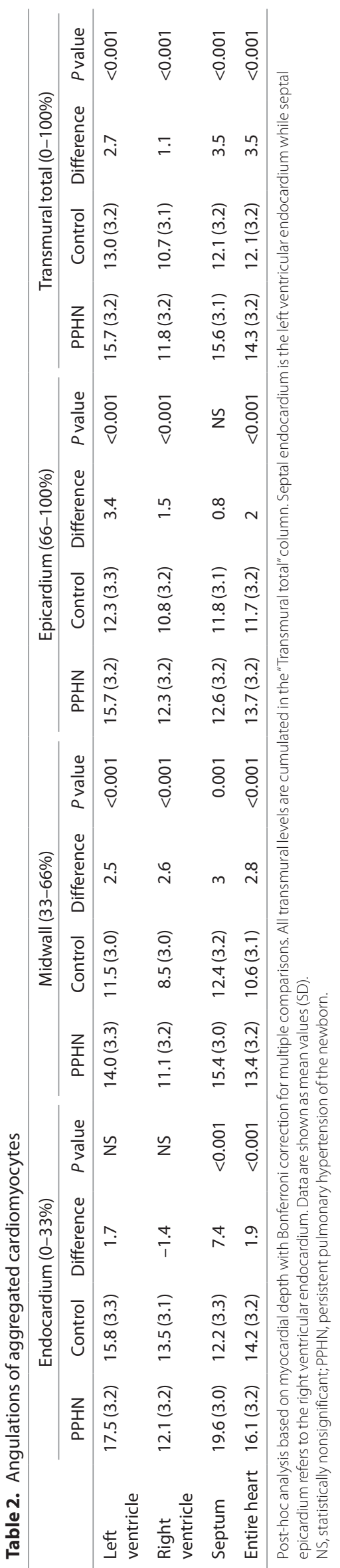

Staining with sirius red failed to reveal any connective tissue separating the superficial layer from the remainder of the myocardium. Judging from the diffusion-weighted images, the epicardial layer of longitudinally oriented cardiomyocytes encompasses the entirety of the right ventricle, and is approximately $1 \mathrm{~mm}$ in thickness. In Figure 5, we show tractographies originating from the zones $2,3,8$, and 9 , and extending to the ventricular septum. The tracts propagate between the septum and the parietal walls of both left and right ventricles, showing that the septum is shared between the ventricles in both normal and hypertensive hearts.

\section{DISCUSSION}

To our knowledge, ours is the first study using diffusion tensor magnetic resonance imaging to provide a morphological investigation of hearts from animals with in-utero pulmonary hypertension mimicking persistent pulmonary hypertension of the newborn. As far as we are aware, it is also the first attempt to quantify angulations of aggregated cardiomyocytes in the right ventricle. We found that the hypertensive hearts have undergone universal hypertrophy, as seen by the significant increase in biventricular myocardial volumes, with maintained cavitary volumes. Overall, ventricular mural thickness was increased throughout the hearts, but only the septal thickening proved to be statistically significant. We found no significant differences in ventricular cavitary volumes, despite significant enlargement of the tricuspid annulus, the latter indicating a tendency toward right ventricular dilation. Others also found an increased overall heart weight using the same ovine model, but with the right ventricle accounting for a larger proportion of global increase than the left ventricle, with reverse findings in normal hearts (12).

\section{Angulations of Cardiomyocytes}

We found that the cardiomyocytes aggregated together within the myocardium showed identical helical and transmural angulations in the hypertensive and control hearts. The difference we found in orientation reflected the angulation of the units into which the individual cardiomyocytes are aggregated. It has been proposed for decades that cleavage planes must exist within the myocardium (13), such planes being of paramount importance for the continuous remodeling and displacement of the aggregated units of cardiomyocytes during the cardiac cycle (14). Rearrangement of the units, or so-called sheets, is needed to explain the phenomenon of systolic myocardial thickening $(14,15)$. Changes in angulations have been found through the cardiac cycle in both rats and humans, adding weight to the notion that aggregation into units is needed for optimal and continuous repacking and rearrangement through the cardiac cycle $(11,16)$. Inappropriate repacking, therefore, could well play a significant role in pathology. One could speculate that a mismatch between the possible change in angulation and the needed mural thickening and repacking might result in poor cardiac function and maybe even heart failure. This notion, however, still remains to be investigated scientifically.

In our data, the mean angle of the aggregated cardiomyocytes was $3.5^{\circ}$ larger in the hypertensive animals as compared 

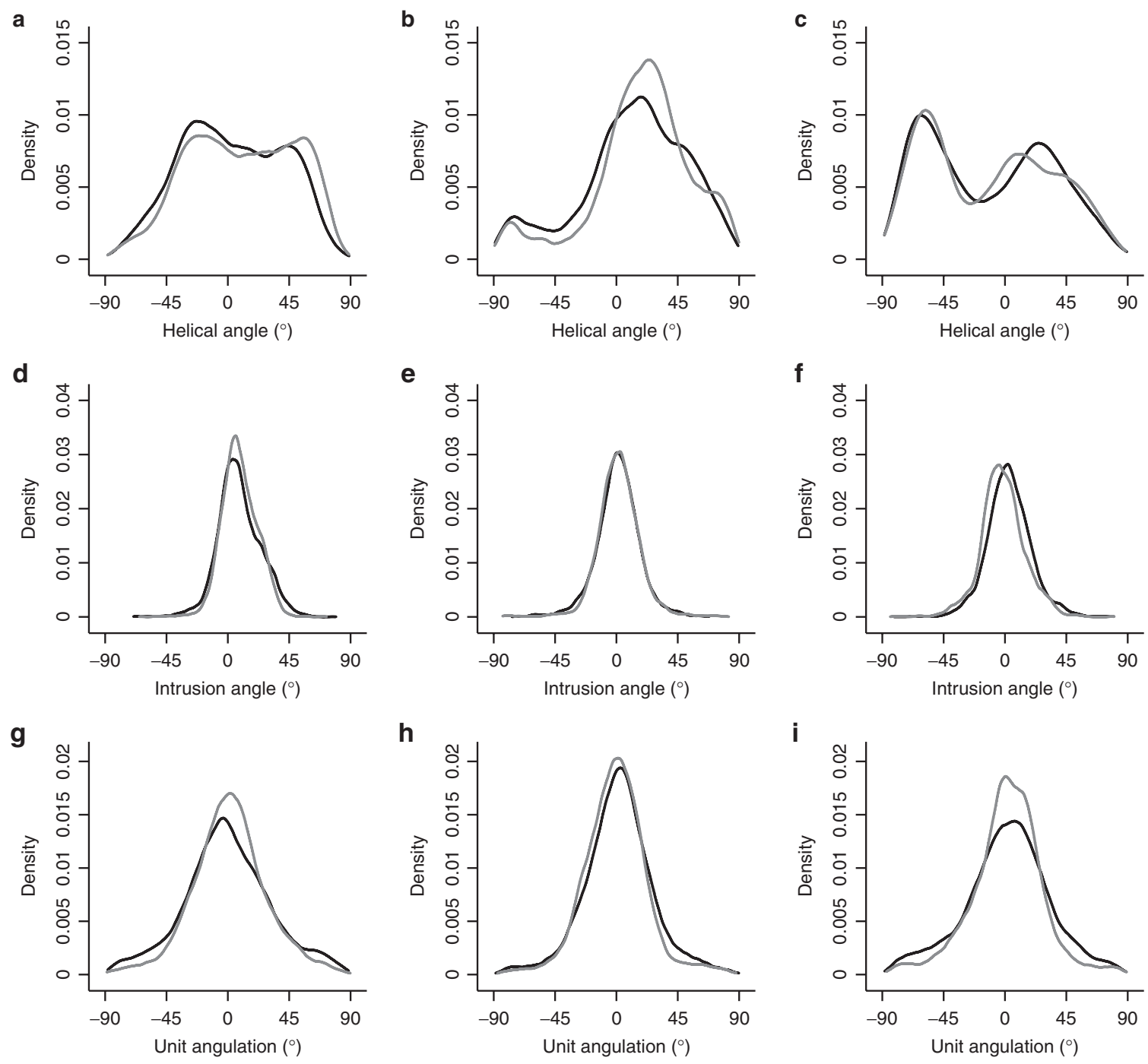

Figure 1. Histograms of the distributions of myocyte angulations. Top panels: helical angles in left ventricle (a), right ventricle (b) and septum (c). Middle panels: intrusion angles in left ventricle (d), right ventricle (e) and septum (f). Bottom panels: unit angulation, often referred to as the sheet angle, in the left ventricle (g), right ventricle (h) and the septum (i). Black lines represent the pulmonary hypertension group while grey represents controls.

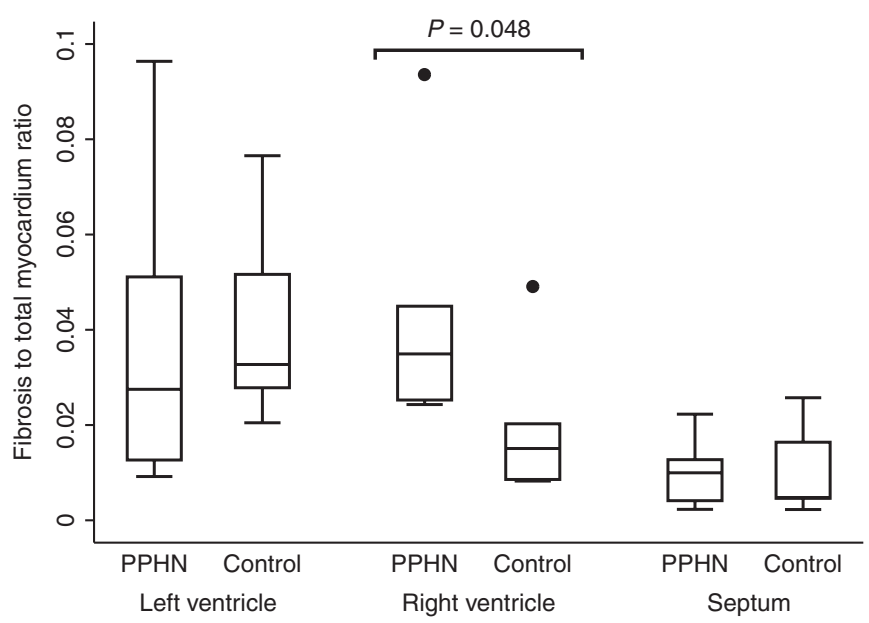

Figure 2. Fibrosis quantification. The fibrosis to myocardium ratio in in-utero pulmonary hypertension ( $n=6)$ vs. controls $(n=5)$. Black dots are outliers. with their controls. This indicates that the units of aggregated cardiomyocytes in the hypertensive lambs, in general, are more parallel to the epicardium than in controls as seen in the normal diastole $(11,16)$, despite being hypertrophied with a tendency toward greater mural thickness. It can be argued that the change in angulation of the aggregated units could also be caused by pathological dilation of the ventricles, reflecting a contractile state more similar to diastole. It must also be kept in mind that the hypertensive hearts by chance could be fixed in a more diastolic state than the controls. Unfortunately, we are not able to test this hypothesis, because ventricular volumes were not measured in vivo in this study. When judging by the appearance of Figure 1, the changes were most obvious in the septum which was the only region where significant wall thickening was found. In the right ventricle, we found signs of ventricular dilation, but the increase in mean angle of the aggregated right ventricular cardiomyocytes was just $1.1^{\circ}$. 
a

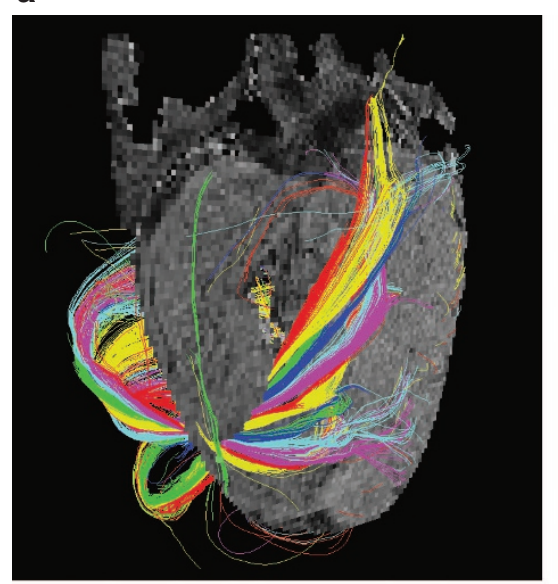

c

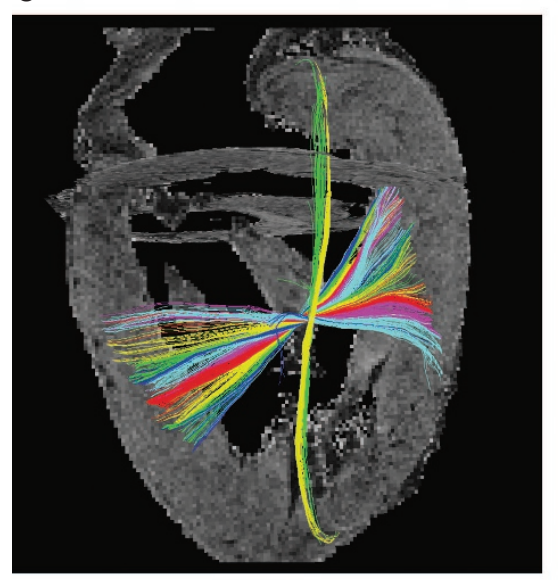

e

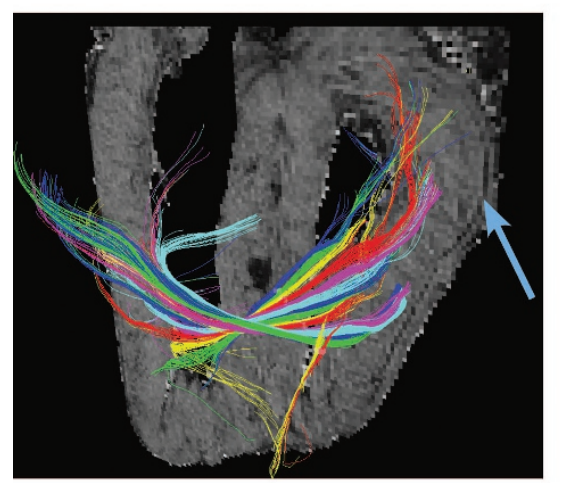

b

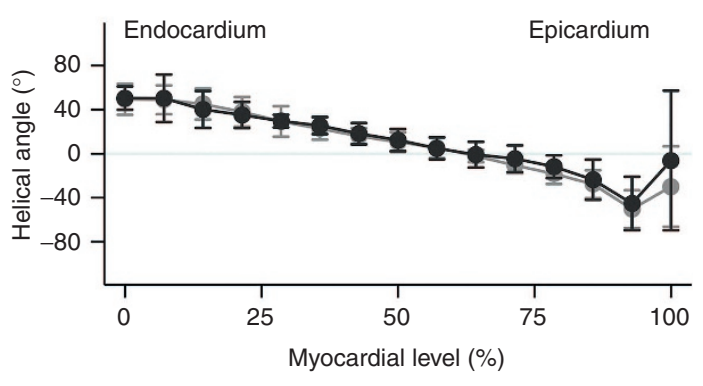

d

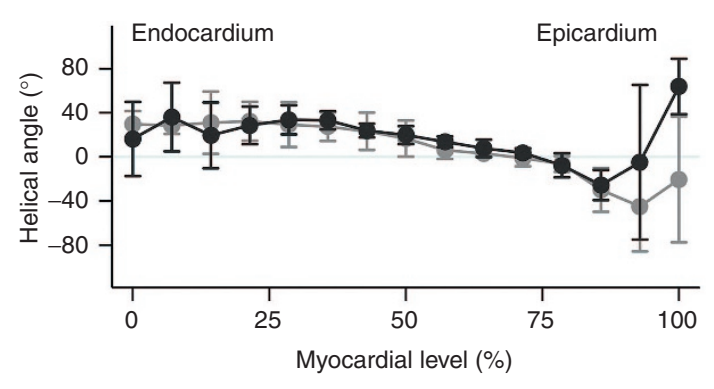

f

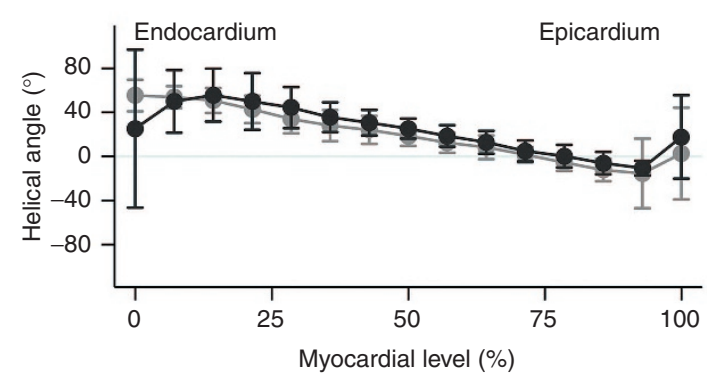

Figure 3. Myocyte orientation in the right ventricle. The superficial "layer" of longitudinal myocytes found in the right ventricle of all hearts here exemplified with tractographies from zones $\mathrm{r} 4$ (a) and $\mathrm{r} 6$ (e) from control hearts, whilst $\mathrm{r} 5$ (c) is from a heart with pulmonary hypertension. Notice the dark line in the background images delineating the crisp transition between the superficial layer and the remaining myocardium (blue arrow). Corresponding plots of helical angles are shown (b, d and f) showing in-utero pulmonary hypertension (grey) vs. controls (black), whiskers outline $95 \% \mathrm{Cl}$. The colour coding of the tractography does not represent any anatomical or physiological property, it only serves as a visual aid enabling the reader to distinguish between the tracks and visualize their propagation. $\mathrm{Cl}$, confidence interval.

\section{Myocardial Fibrosis}

Myocardial fibrosis is well known in the setting of pulmonary arterial hypertension $(17,18)$. The presence of myocardial fibrosis in patients with persistent pulmonary hypertension in the newborn, in contrast, is not well described. This disease usually has a rather acute onset as compared with the more chronic disorder of pulmonary arterial hypertension, hence presence of fibrosis in the newborn with pulmonary hypertension is not a matter of course. Nonetheless, our data confirm the presence of excess collagen in the right ventricular myocardium in the setting of in-utero pulmonary hypertension. In fact, the amount of collagen in the investigated zone of the right 


\section{Articles | Agger et al.}
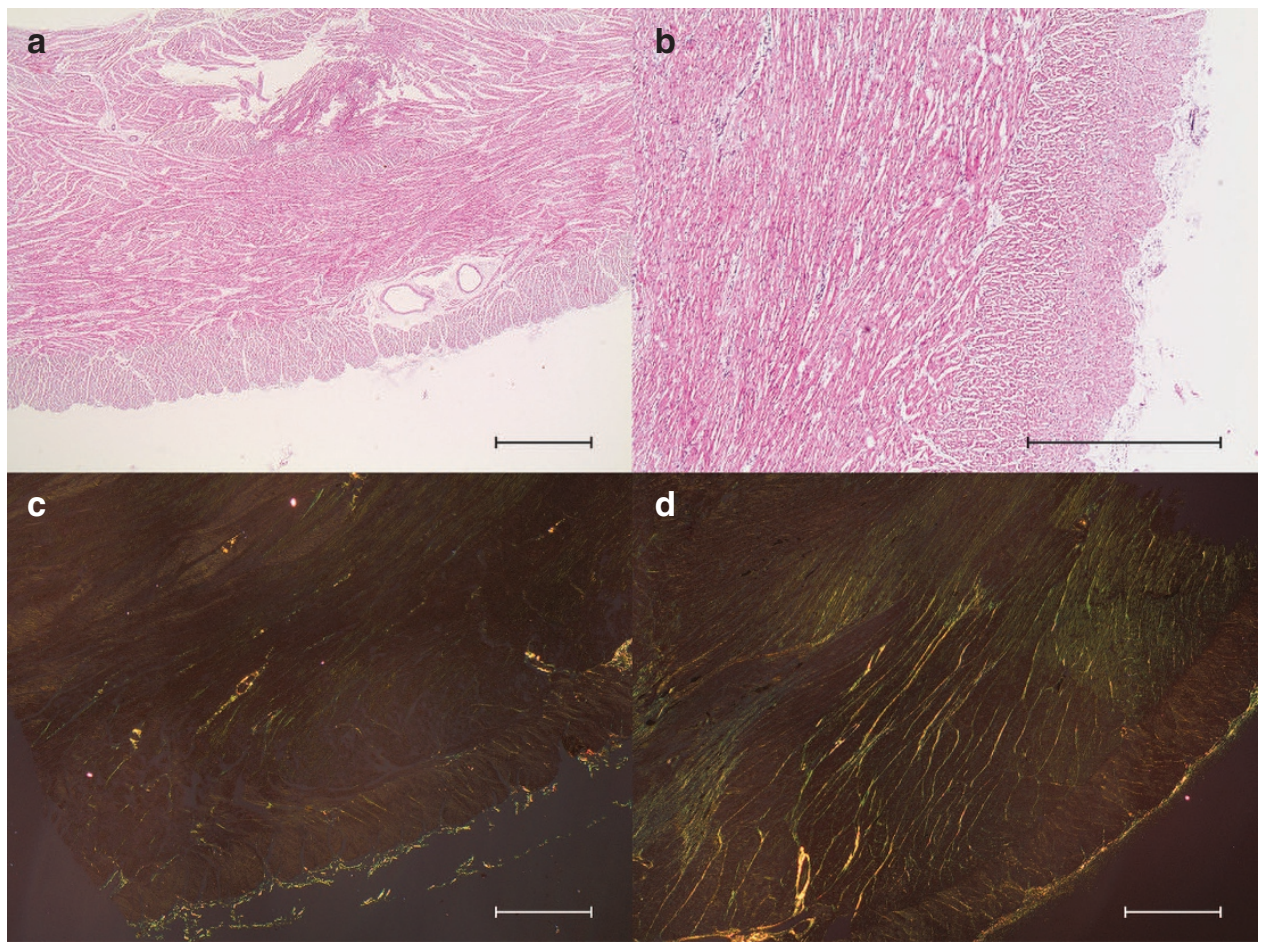

Figure 4. Histological investigations. Histological sections of the right ventricle in controls (a and $\mathbf{c}$ ) and pulmonary hypertensive lambs (b and $\mathbf{d})$. All sections show the epicardial layer of longitudinally oriented cardiomyocytes distinct relative to the deeper myocardium. Overall histology is shown in panels $\mathbf{a}$ and $\mathbf{b}$ using haematoxylin-eosin staining, while collagen is coloured in panels $\mathbf{c}$ and $\mathbf{d}$ using sirius red staining in polarized light. A complete figure of all biopsies stained with sirius red in polarized light is provided as supplemental material (see Supplementary Figure $\mathbf{S 1}$ online). Bar = $1 \mathrm{~mm}$.

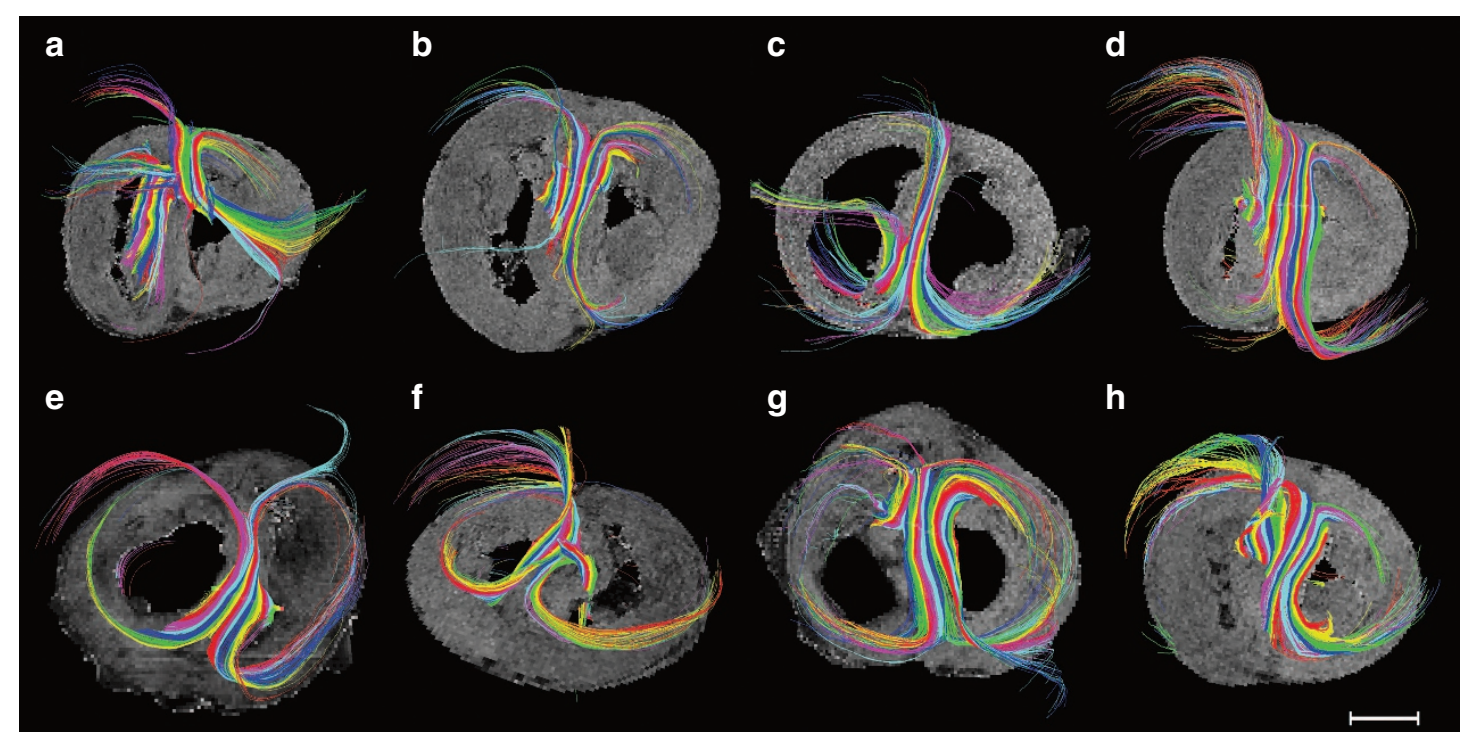

Figure 5. Myocyte orientation in the interventricular septum. The tractography shows how the septum is "shared" between the left and the right ventricles. Hearts with pulmonary hypertension are shown in the top panels (a through $\mathbf{d}$ ), while the bottom panels show hearts from controls (e through $\mathbf{h}$ ). By comparing the panels vertically the sharing in different zones can be compared between groups: zone 2 ( $\mathbf{a}$ and $\mathbf{e}$ ), zone 3 (b and $\mathbf{f}$ ), zone 8 (c and $\mathbf{g})$ and zone 9 (d and $\mathbf{h})$. Bar $=1 \mathrm{~cm}$.

ventricle is more than doubled relative to controls. A fibrosis area of $3.8 \%$ is rather small compared with what is found in adults with other types of pulmonary hypertension (19). This could be due to the relatively short duration of the disease in this experimental model or it could be an inherent feature of persistent pulmonary hypertension in the newborn. Due to the lack of literature on the histological findings of this disease, we are unable to answer this question. The notion has already been proposed that an increase in myocardial fibrosis might hamper the ability of the units of aggregated cardiomyocytes, whatever their dimensions and alignments, to slide against one another, thus leading to heart failure (20). We investigated 
the correlation between the fibrosis ratio and the angle of the aggregated myocytes, but were unable to detect a correlation between the two. Thus, we cannot prove the change in angulation of the aggregated cardiomyocytes to be caused by fibrosis.

It may very well be speculated that if myocardial fibrosis is a significant contributor to the pathology of persistent pulmonary hypertension in the newborn then the use of conventional inotropes for the treatment of heart failure in this condition may have to be reevaluated. We cannot provide sufficient scientific evidence in this study to offer solid conclusions in this matter, but clearly further investigations are indicated.

\section{Longitudinally Oriented Right Ventricular Myocytes}

To our knowledge, the superficial layer of longitudinal myocytes in the right ventricle has never previously been documented in neither persistent pulmonary hypertension in the newborn nor in normal mammalian hearts in general. The finding gives rise to several questions. What is its purpose? Is it a neonatal or an ovine phenomenon? Does it disappear with age? Why is there no similar layer to be found in the left ventricle? We accept that it is a gross simplification to describe "layers" as such in the myocardium, since no fibrous septae are to be found between the supposed layers (21). The epicardial layer found in the right ventricles of the hearts of our study, nonetheless, is indeed a true layer, with a clear break in angulation relative to the deeper cardiomyocytes, even though no fibrous insulation was found at the transitional level. Longitudinal orientation of right ventricular cardiomyocytes in the endocardium is a well-described phenomenon (22), but we could find no previous descriptions of such an epicardial longitudinal layer, in neither neonates nor adults of any species. We suspect that if such epicardial longitudinal layer was previously described in adults, it's existence would be common knowledge. Hence, we consider it most likely to be a fetal phenomenon, which most likely will disappear as a part of the adaptation from the fetal to the postnatal circulation. The vast majority of the myocytes in the right ventricle is oriented in near circumferential fashion (22). Contraction of these myocytes will not only mainly serve to generate pressure but will also cause ventricular elongation, which is not beneficial for ejection and maintenance of the relatively high fetal right ventricular pressure. The right ventricular epicardial layer of longitudinally oriented myocytes may thus serve as to counteract this elongation and thereby improve the pressure generation possible for the right ventricle.

Mammalian hearts have very few overall structural differences $(23,24)$. It is unlikely, therefore, that the neonatal sheep should be any different from other mammals. Human hearts ranging from 13 gestational weeks to 90 years of age have been studied carefully, largely using dissections, but supplemented with histological evaluations (25). The developing porcine heart has similarly been studied, using diffusion tensor magnetic resonance imaging supplemented with histological examinations (26). Neither of these studies mentions a superficial layer comparable to our finding
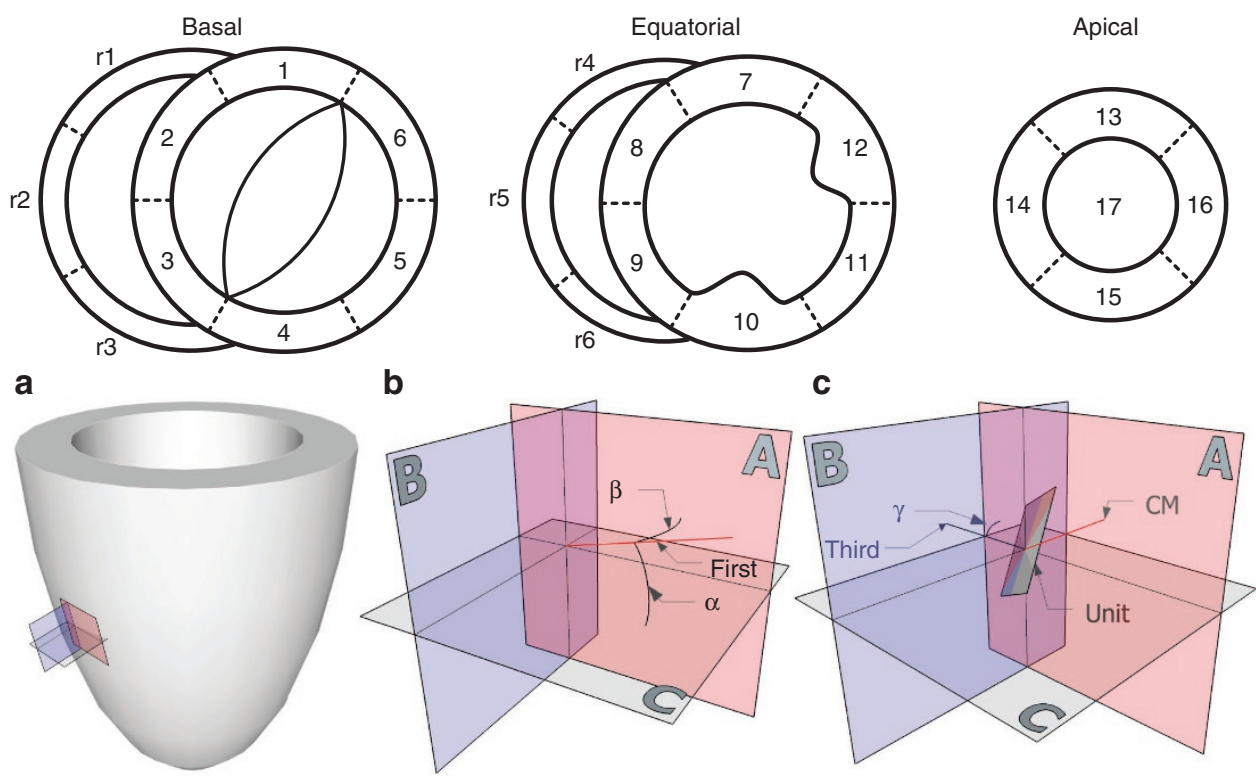

Figure 6. Myocardial zones and angle definitions. Top panels: Schematic representation of the cardiac zones adapted and further developed from Cerqueira (35) and coworkers. Left ventricle: 1, basal superior; 2 , basal superoseptal; 3 , basal inferoseptal; 4, basal inferior; 5 , basal inferolateral; 6, basal superolateral; 7 , equatorial superior; 8 , equatorial superoseptal; 9 , equatorial inferoseptal; 10 , equatorial inferior; 11 , equatorial inferolateral; 12 , equatorial superolateral; 13 , apical superior; 14, apical septal; 15, apical inferior; 16, apical lateral; 17, apex. Right ventricle: $r 1$, basal anterosuperior; $r 2$, basal anterior; $r 3$, basal anteroinferior; $r 4$, equatorial anterosuperior; $r 5$, equatorial anterior; $r 6$, equatorial anteroinferior. $\mathbf{a}$ : The left ventricle with the local orthogonal planes aligned relative to the epicardium. $\mathbf{b}$ : The local set of orthogonal planes as superimposed in every voxel. Plane $A$ is the circumferential-longitudinal plane parallel to the epicardial tangential plane and plane B is the radial-longitudinal plane parallel to the left ventricular long axis and orthogonal to plane A. Plane C, the local "horizontal" plane, is the circumferential-radial plane orthogonal to planes A and B. The helical angle $\alpha$ is the angle between the primary eigenvector (first) and plane C. The transmural angle $\beta$ is the angle between the primary eigenvector and plane A. c: The unit angle $\gamma$ is measured using the tertiary eigenvector (third) relative to the circumferential-longitudinal plane. The unit of aggregated cardiomyocytes (CM) is depicted as a flat square, which is a gross oversimplification. 
in the right ventricle. Further studies are needed, therefore, to validate whether the epicardial layer of longitudinally oriented myocytes in the right ventricle is a new anatomical substructure present in all neonate mammals, including humans.

\section{Septal Ownership}

It seems to be common wisdom that the interventricular septum should be considered a left ventricular structure. In 1669, for example, Lower (27) noted that "the septum helps the contraction of the left ventricle only" and 200 years later Pettigrew (23) noted that only two-thirds of the septum is of left ventricular origin, whilst the remaining third originates from the right ventricle. Greenbaum and colleagues (21) recognized that the septum was constructed from subendocardial fibers from both ventricles, but concluded that the septum belongs to the left ventricle by virtue of the thick central layer originating solely from the left ventricle. When assessing the origin of the ventricular cardiomyocytes based on genetic markers specific to either the left or the right ventricle, the ventricular septum is found to be built up from cardiomyocytes with both right and left ventricular lineages (28). Furthermore, recent investigations using diffusion tensor imaging showed a comparable change through fetal and postnatal development from equal contributions to left ventricular domination (26).

The sharing of the ventricular septum between the ventricles might be expected to lead to the finding of a distinct circular layer of cardiomyocytes in the hypertrophic right ventricles of our pulmonary hypertensive lambs (29). Our measurements of helical angles militate against the presence of such a distinct collection of circumferentially aligned cardiomyocytes. This is supported by our earlier investigation of isolated right ventricular hypertrophy (8).

The functional and clinical significance of these findings remains unclear. The clinical course of pulmonary hypertension is mainly determined by the dysfunction of the right ventricle (30). The inability of the hypertrophied right ventricle to create a circular middle myocardial layer may suggest that it is more likely to fail and decompensate in the presence of increased afterload as compared with the left ventricle.

In conclusion, we have shown that, in the setting of a model of persistent pulmonary hypertension of the newborn, the heart undergoes significant morphological changes in terms of overall anatomical dimensions and fibrosis. We found evidence of impairment of the ability of the myocardial units to slide one upon the other so as to promote myocardial mural thickening. The pathological basis for this finding remains unclear. We have also demonstrated a right ventricular subepicardial layer of strictly longitudinally oriented cardiomyocytes, which has an abrupt transition to the remaining deeper right ventricular cardiomyocytes. To the best of our knowledge, this layer has not previously attracted comment. Finally, we have added additional evidence to the question of the ventricular ownership of the septum, arguing that the septum is not strictly a left ventricular structure. Further studies evaluating the association between these structural changes and ventricular function during normal and abnormal transition at birth leading to persistent pulmonary hypertension are warranted.

\section{METHODS}

\section{Animal Experiments}

Time-dated pregnant ewes were obtained from the Swartz family farm (Attica, NY). Antenatal ligation of the arterial duct was performed on six lambs at a gestational age of 127 days (full term $\sim 145$ days) to induce pulmonary hypertension, as previously described (31). Five healthy sibling lambs served as controls. All 11 lambs were delivered 8 days later. Lambs were intubated and fetal lung liquid was drained. They were placed under a servo-controlled radiant warmer and ventilated with $100 \% \mathrm{O}_{2}$ (Servo300 ventilator, Siemens, Mississauga, Ontario, Canada), as previously described (32). Umbilical arterial and venous lines were placed. Propofol $(1-5 \mathrm{mg} / \mathrm{kg} / \mathrm{h})$ and fentanyl $(2-4 \mu \mathrm{g} / \mathrm{kg} / \mathrm{h})$ were administered by continuous intravenous infusion. Additional boluses of fentanyl $(5 \mu \mathrm{g} / \mathrm{kg})$ were administered to provide analgesia in the presence of signs of pain and/or discomfort. Intravenous fluids were administered at $100 \mathrm{ml} / \mathrm{kg} /$ day. Ventilator settings were adjusted to maintain $\mathrm{PaCO}_{2}$ between 35 and $50 \mathrm{mmHg}$ guided by arterial blood gases. Lambs were killed with an overdose of thiopental sodium $(100 \mathrm{mg} / \mathrm{kg})$ and exsanguinated. The heart and lungs were removed en bloc through a median sternotomy, and the coronary arteries were flushed with cardioplegic solution $(20 \mathrm{mmol} / \mathrm{l}$ potassium-supplemented Lactated Ringers). The whole heart samples were fixed with $10 \%$ formalin through manual coronary perfusion at subphysiological pressure. The hearts were kept in formalin 2-3 days ensuring complete fixation, rinsed and stored in PBS at $4{ }^{\circ} \mathrm{C}$ until scanning.

\section{Imaging Sequence}

Examinations were performed with a 9.4-T preclinical MRI system (Agilent, Santa Clara, CA), equipped with a 400-mT gradient system and vnmrJ 4.0 operating system. Room temperature was maintained constant at $22.0 \pm 1.5^{\circ} \mathrm{C}$ and humidity at $50 \pm 10 \%$. A diffusion tensor magnetic resonance sequence was employed with a multislice $2 \mathrm{D}$ spin-echo sequence with an isotropic voxel resolution of $310 \times 310 \times 310 \mu \mathrm{m}^{3}$, repetition time: 7,000 ms, echo time: $30 \mathrm{~ms}$. One single repetition of diffusion sensitive images with 30 diffusion encoded directions isotropically distributed was acquired (33) with diffusion sensitivity $b$ values of $1,000 \mathrm{~s} / \mathrm{mm}^{2}$ and one image with $b$ value of $0 \mathrm{~s} / \mathrm{mm}^{2}$. Total scanning time was approximately 16 hours per heart depending on individual size. Assuming an average volume of a cardiomyocyte of 32 picolitres (34) each voxel represents the average direction of $\sim 900$ myocytes.

\section{Anatomical Measurements}

The ventricular mass was divided into 17 left ventricular zones, and 6 right ventricular zones, expanding an already well-established concept (35) to include the right ventricle (Figure 6). We took no measurements from zones $1,4,10,12$, and 17 so as to avoid conflicting issues such as papillary muscles, ventricular hinge points, and the apical vortex. We measured both myocardial and ventricular volumes by segmentation of the diffusion-weighted images, using the atrioventricular and ventriculoarterial valvar leaflets to delimit the ventricular volumes. For each zone, we measured mural thickness perpendicular to the epicardial surface.

\section{Myocardial Fibrosis}

After magnetic resonance examination, the hearts were cut in the short axis plane at the equatorial level. Transmural biopsies were cut from zone \#11 in the left, zone \#r5 in the right ventricle, and from zone \#8 in the septum. The origin of the biopsies was blinded to all investigators through the remainder of the assessment. The biopsies were prepared for histological examination and stained with sirius red. The entire cut face of each biopsy was photographed, using $2 \times$ magnification, in linear polarized light. The angulation of the polarized light and the exposure time were maintained identical in all photographs. Subsequently, the images were merged using user independent functions in Adobe Photoshop CS6 (Adobe Systems 
Software, Ireland). The area of collagen was assessed selecting the yellow colors using the investigator independent "Color Range" tool in Photoshop. Vessels, along with the epi- and endocardium, being high in natural collagen content, were excluded from the selection. The area occupied by collagen was then compared with the total area of the biopsy, providing the ratio of fibrosis. In case of significant difference within a region, post-hoc analysis was done by dividing the region into three transmural sections, each covering one-third of the transmural thickness.

\section{Measurements of Myocardial Architecture}

The diffusion data were imported into our custom-made analysis software as used in previous contributions from our group $(8,9,36)$. Within each zone, the three eigenvectors of the diffusion tensor were exported from a transmural region of interest measuring $3 \times 3$ voxels. Within each voxel, we superimposed a local coordinate system and assessed the helical and transmural angulations as outlined in Figure 6. The superimposed local planes are introduced, recognizing the importance of measuring relative to the epicardial tangential plane. The rearrangement of cardiomyocytes through the cardiac cycle, in other words systolic mural thickening, takes place relative to the epicardial tangential plane. We must assume, therefore, that angulations must be measured relative to that plane to provide the most relevant information on anatomy, and especially function. Within each zone, the myocardial architecture was evaluated qualitatively using transmural FACT tractography (37). The software was allowed to track through primary eigenvectors with a fractional anisotropy threshold of 0.15 and an inner product threshold of 0.75 . These thresholds are commonly used in literature $(38,39)$. Depending on the area of interest, the hearts were evaluated with different maximum track-length thresholds from 30 to $100 \mathrm{~mm}$.

\section{Statistical Analyses}

Normality was confirmed in all primary endpoints using histograms and Q-Q plots. Overall analysis of mural thickness was done in each ventricle by repeated measures ANOVA, while changes in mural architecture was tested between groups using a nested ANOVA model. Results are reported as means followed by SD in parentheses. Post-hoc analyses were done using Student's $t$-test. All tests presumed a significance level of 5\%. Stata Statistical Software, release 11 (StataCorp LP, TX) was used for all statistical analyses.

\section{Heuristic Considerations}

The extent, and appearance, of the aggregated cardiomyocytes has yet to be clarified. It has been suggested that transmural sheets exist due to aggregation of four to six cardiomyocytes (40). This is probably a gross oversimplification, but has been widely accepted as a fact. The extent of the remaining two dimensions of any aggregated entities, nonetheless, is even more difficult to assess, and remains a topic of intense discussion. In short, the anatomical appearance of what may be considered "sheets", or "lamellar units", remains unclear. Because of this, we have simply described units of aggregated cardiomyocytes, remembering that the phenomenon we wish to quantify is still not a well-described anatomical entity. To date, furthermore, there is no general agreement on how to interpret the angulation of the units produced by the aggregation in a physiological fashion. Helical and transmural angles are angulations of the chains of cardiomyocytes themselves, whilst the angulation of the aggregated cardiomyocytes takes into account the three-dimensional orientation of the adjacent myocytes aggregated together. Only the tertiary eigenvector truly serves the purpose of describing the three-dimensional orientation of the aggregated since only this vector assumes the direction of the normal of the unit plane. This depends, of course, on the assumptions that the unit is some sort of flattened structure and that the cardiomyocytes are aligned parallel to the surface of the aggregated units. This is supported by images produced using scanning electron microscopy (20).

\section{Ethics}

This animal protocol was approved by the Laboratory Animal Care Committees at the University at Buffalo, Buffalo, NY, USA, and Northwestern University, Chicago, IL, USA.

\section{SUPPLEMENTARY MATERIAL}

Supplementary material is linked to the online version of the paper at http:// www.nature.com/pr

\section{ACKNOWLEDGMENTS}

The authors thank Anette Funder at the Department of Forensics, Aarhus University Hospital, Denmark for preparing and staining the histological slides.

\section{STATEMENT OF FINANCIAL SUPPORT}

This work was supported by the Danish Children's Heart Foundation (P.A.); The Arvid Nilsson Foundation, Denmark (P.A.); and the National Heart Lung and Blood Institute, USA [NHLBI L54705] (R.H.S.).

Disclosures: None.

\section{REFERENCES}

1. Nair J, Lakshminrusimha S. Update on PPHN: mechanisms and treatment. Semin Perinatol 2014;38:78-91.

2. Steinhorn RH. Diagnosis and treatment of pulmonary hypertension in infancy. Early Hum Dev 2013;89:865-74.

3. Stayer SA, Liu Y. Pulmonary hypertension of the newborn. Best Pract Res Clin Anaesthesiol 2010;24:375-86.

4. St John Sutton MG, Meyer RA. Left ventricular function in persistent pulmonary hypertension of the newborn. Computer analysis of the echocardiogram. Br Heart J 1983;50:540-9.

5. Riemenscneider TA, Nielsen HC, Ruttenberg HD, Jaffe RB. Disturbances of the transitional circulation: spectrum of pulmonary hypertension and myocardial dysfunction. J Pediatr 1976;89:622-5.

6. Scollan DF, Holmes A, Winslow R, Forder J. Histological validation of myocardial microstructure obtained from diffusion tensor magnetic resonance imaging. Am J Physiol 1998;275:H2308-18.

7. Mekkaoui $\mathrm{C}$, Huang $\mathrm{S}$, Chen $\mathrm{HH}$, et al. Fiber architecture in remodeled myocardium revealed with a quantitative diffusion CMR tractography framework and histological validation. J Cardiovasc Magn Reson 2012;14:70.

8. Nielsen E, Smerup M, Agger P, et al. Normal right ventricular threedimensional architecture, as assessed with diffusion tensor magnetic resonance imaging, is preserved during experimentally induced right ventricular hypertrophy. Anat Rec (Hoboken) 2009;292:640-51.

9. Smerup M, Agger P, Nielsen EA, et al. Regional and epi- to endocardial differences in transmural angles of left ventricular cardiomyocytes measured in ex vivo pig hearts: functional implications. Anat Rec (Hoboken) 2013;296:1724-34.

10. Streeter DD Jr, Spotnitz HM, Patel DP, Ross J Jr, Sonnenblick EH. Fiber orientation in the canine left ventricle during diastole and systole. Circ Res 1969;24:339-47.

11. Ferreira PF, Kilner PJ, McGill LA, et al. In vivo cardiovascular magnetic resonance diffusion tensor imaging shows evidence of abnormal myocardial laminar orientations and mobility in hypertrophic cardiomyopathy. J Cardiovasc Magn Reson 2014;16:87.

12. Belik J, Halayko AJ, Rao K, Stephens NL. Fetal ductus arteriosus ligation. Pulmonary vascular smooth muscle biochemical and mechanical changes. Circ Res 1993;72:588-96.

13. Mall FP. On the muscular architecture of the ventricles of the human heart. Am J Anat 1911;11:211-66.

14. Spotnitz HM, Spotnitz WD, Cottrell TS, Spiro D, Sonnenblick EH. Cellular basis for volume related wall thickness changes in the rat left ventricle. J Mol Cell Cardiol 1974;6:317-31.

15. Waldman LK, Nosan D, Villarreal F, Covell JW. Relation between transmural deformation and local myofiber direction in canine left ventricle. Circ Res 1988;63:550-62.

16. Hales PW, Schneider JE, Burton RA, Wright BJ, Bollensdorff C, Kohl P. Histo-anatomical structure of the living isolated rat heart in two contraction states assessed by diffusion tensor MRI. Prog Biophys Mol Biol 2012;110:319-30.

17. Ryan JJ, Archer SL. The right ventricle in pulmonary arterial hypertension: disorders of metabolism, angiogenesis and adrenergic signaling in right ventricular failure. Circ Res 2014;115:176-88. 
18. Naeije R, Manes A. The right ventricle in pulmonary arterial hypertension. Eur Respir Rev 2014;23:476-87.

19. Rain S, Handoko ML, Trip P, et al. Right ventricular diastolic impairment in patients with pulmonary arterial hypertension. Circulation 2013;128:2016-25, 1-10.

20. Lunkenheimer PP, Niederer P. Hierarchy and inhomogeneity in the systematic structure of the mammalian myocardium: towards a comprehensive view of cardiodynamics. Technol Health Care 2012;20:423-34.

21. Greenbaum RA, Ho SY, Gibson DG, Becker AE, Anderson RH. Left ventricular fibre architecture in man. Br Heart J 1981;45:248-63.

22. Ho SY, Nihoyannopoulos P. Anatomy, echocardiography, and normal right ventricular dimensions. Heart 2006;92(Suppl 1):i2-13.

23. Pettigrew JB. On the arrangement of the muscular fibres in the ventricles of the vertebrate heart, with physiological remarks. Philos Trans R Soc Lond A 1864;154:445-505.

24. Grimm AF, Katele KV, Lin HL. Fiber bundle direction in the mammalian heart. An extension of the "nested shells" model. Basic Res Cardiol 1976;71:381-8.

25. Sanchez-Quintana D, Garcia-Martinez V, Climent V, Hurle JM. Morphological changes in the normal pattern of ventricular myoarchitecture in the developing human heart. Anat Rec 1995;243:483-95.

26. Zhang L, Allen J, Hu L, Caruthers SD, Wickline SA, Chen J. Cardiomyocyte architectural plasticity in fetal, neonatal, and adult pig hearts delineated with diffusion tensor MRI. Am J Physiol Heart Circ Physiol 2013;304:H246-52.

27. Lower R. Tractatus de Corde. London, United Kingdom: Early science in Oxford, 1669.

28. Franco D, Meilhac SM, Christoffels VM, Kispert A, Buckingham M, Kelly RG. Left and right ventricular contributions to the formation of the interventricular septum in the mouse heart. Dev Biol 2006;294: 366-75.

29. Sanchez-Quintana D, Anderson RH, Ho SY. Ventricular myoarchitecture in tetralogy of Fallot. Heart 1996;76:280-6.
30. Singh GK, Levy PT, Holland MR, Hamvas A. Novel methods for assessment of right heart structure and function in pulmonary hypertension. Clin Perinatol 2012;39:685-701.

31. Morin FC 3rd, Egan EA. The effect of closing the ductus arteriosus on the pulmonary circulation of the fetal sheep. J Dev Physiol 1989;11:283-7.

32. Lakshminrusimha S, Russell JA, Wedgwood S, et al. Superoxide dismutase improves oxygenation and reduces oxidation in neonatal pulmonary hypertension. Am J Respir Crit Care Med 2006;174:1370-7.

33. Jones DK. The effect of gradient sampling schemes on measures derived from diffusion tensor MRI: a Monte Carlo study. Magn Reson Med 2004;51:807-15.

34. Satoh H, Delbridge LM, Blatter LA, Bers DM. Surface:volume relationship in cardiac myocytes studied with confocal microscopy and membrane capacitance measurements: species-dependence and developmental effects. Biophys J 1996;70:1494-504.

35. Cerqueira MD, Weissman NJ, Dilsizian V, et al.; American Heart Association Writing Group on Myocardial Segmentation and Registration for Cardiac Imaging. Standardized myocardial segmentation and nomenclature for tomographic imaging of the heart. A statement for healthcare professionals from the Cardiac Imaging Committee of the Council on Clinical Cardiology of the American Heart Association. Circulation 2002;105:539-42.

36. Smerup M, Nielsen E, Agger P, et al. The three-dimensional arrangement of the myocytes aggregated together within the mammalian ventricular myocardium. Anat Rec (Hoboken) 2009;292:1-11.

37. Mori S, Crain BJ, Chacko VP, van Zijl PC. Three-dimensional tracking of axonal projections in the brain by magnetic resonance imaging. Ann Neurol 1999;45:265-9.

38. Wakana S, Jiang H, Nagae-Poetscher LM, van Zijl PC, Mori S. Fiber tractbased atlas of human white matter anatomy. Radiology 2004;230:77-87.

39. Rollins N, Reyes T, Chia J. Diffusion tensor imaging in lissencephaly. AJNR Am J Neuroradiol 2005;26:1583-6.

40. Caulfield JB, Borg TK. The collagen network of the heart. Lab Invest 1979;40:364-72. 